\title{
Drug Hypersensitivity Reduction Using Encapsulation Method with Chitosan-Cetirizine Derivatives
}

\author{
IONUT DRAGOSTIN ${ }^{1}$, OANA MARIA DRAGOSTIN²*, MARIA DRAGAN³, CATALINA DANIELA STAN³, \\ CARMEN LACRAMIOARA ZAMFIR ${ }^{1}$ \\ ${ }^{1}$ University of Medicine and Pharmacy Grigore T. Popa, Faculty of Medicine, Department of Histology, 16 Universitatii Str., 700115, \\ Iasi, Romania; \\ 2University Dunarea de Jos, Faculty of Medicine and Pharmacy, Research Centre in the Medical-Pharmaceutical Field,47 \\ Domneasca Str., 800008, Galati, Romania; \\ 3University of Medicine and Pharmacy Grigore T. Popa, Faculty of Pharmacy, Department of Drug Industry and Pharmaceutical \\ Biotechnology, 16 Universitatii Str., 700115, Iasi, Romania.
}

\begin{abstract}
The ideal material for encapsulation should have good rheological properties, good dispersing capacity of active substances, lack of chemical reactivity with encapsulated substances, the ability to complete release the active compounds, under different conditions, solubility in non-toxic solvents. The degree of novelty of this work consists in applying a preclinical solution for reducing the risk of allergic reaction manifestation, after the treatment with therapeutic agents with allergic potential, as it is the case of beta-lactam antibiotics or non-steroidal anti-inflammatory drugs. The originality of this research it is based on obtaining new chitosan derivatives, starting from cetirizine which is well known for its antihistaminic properties, followed by the use of these new derivatives for encapsulation of drugs with allergic potential. The obtained particles will lead to the reducing of the drug hypersensitivity, keeping, at the same time, the followed therapeutic effect.
\end{abstract}

Keywords: chitosan-cetirizine, encapsulation, drug hypersensitivity, microparticles

Antihistamines inhibit the effects of histamine by blocking $\mathrm{H} 1$ receptors and have numerous clinical indications such as: allergic pathologies (rhinitis, atopic dermatitis, allergic conjunctivitis, drug hypersensitivity, urticaria, transfusion reactions), idiopathic chronic urticaria, motion sickness, vertigo and insomnia [1, 2]. Cetirizine, an important representative of this class, is a potent antihistamine $\mathrm{H} 1$ of the second generation, a hydroxysin metabolite, effective in the treatment of allergic rhinitis, chronic urticaria, pollen induced asthma and atopic dermatitis. It becomes differentiated from traditional antagonists by not producing drowsiness or anticholinergic effects $[3,4]$.

On the other hand, aspirin, the most used drug in the world (especially for the prevention of cardiovascular disease, but also in acute inflammation, myalgia, fever, angina or thrombosis), has some severe side effects such as prolonging bleeding time, gastric irritability, and favoring ulceration, as well as inducing hypersensitivity in some subjects. In addition, penicillin and penicillin derivatives such as amoxicillin or ampicillin may also cause acute or subacute allergic reactions mediated by immunoglobulins E and $G[5,6]$.

Microencapsulation of active substances is of major interestin the controlled release range, due to the relatively light microparticle design and reproducibility of the process[7-9]. The importance of biopolymers (chitosan type) is made evident by a multitude of studies, that have highlighted over the time, their characteristics such as: inertness and compatibility with drug substances, nontoxic, protective, antioxidant and biodegradable properties, but also the possibility of creating controlled release systems of active substances [10]. Thus, using chemically modified chitosan via cetirizine, as a polymer matrix, this study attempted to prepare microparticles incorporating aspirin and penicillin, substances well known for their highly allergenic properties. The present work purpose is the development and the validation of some micro-systems based on natural polymers, type of chitosan and its chitosan-cetirizine derivatives, for reducing the drug hypersensitivity concerning the patients with extreme sensitivity to a particular active substance. For this, the following research directions will be pursued: (i) the synthesis, characterization and physico-chemical and spectral (infrared spectroscopy) evaluation of certain derivatives of chitosan-cetirizine (using chitosan with low molecular weight-CLMW and medium molecular weight -CMMW); (ii) the preparation of microparticles based on these derivatives, in which substances with potential allergens were included: aspirin and penicillin; (iii) the morphological characterization of the obtained microparticles using the electronic scanning microscopy (SEM) and, last but not least, (iv) determining the swelling capacity of the obtained polymer matrices, and ( $V$ ) assessing their biodegradability.

\section{Experimental part}

Materials and Methods

General procedure for obtaining chitosan-cetirizine derivatives

The study will mainly focus on the synthesis methods that are based on the principles of green chemistry with the purpose of using them to obtain chitosan derivatives with anti-allergic properties [11-13].

$1 \mathrm{~g}$ of cetirizine ( 0.0025 moles) was subjected to the esterification reaction by mechanical agitation with $2 \%$ chitosan solutions (CLMW and CMMW) in $2 \%$ acetic acid, for 24 hours, using an acid medium (conc. $\mathrm{H}_{2} \mathrm{SO}_{4}$ ), as dehydrating agent. At the end of the reaction time, the products (1, 2- fig. 1) were separated by filtration, the technique being taken from the literature, with minor modifications $[14,15]$. 
IR spectral analysis of chitosan-cetirizine derivatives: structural confirmation

The spectra of the synthesized compounds were performed by ATR-FTIR measurements using FT-IR ABB Bomem MB-3000 (Canada) spectrometer. The spectra were recorded in the range of $4000-500 \mathrm{~cm}^{-1}$ with 32 scans at a resolution of $4 \mathrm{~cm}^{-1}$. Spectral processing was carried outby means of a Horizon MBTM FTIR Software and GRAMS 32 Software (Galactic Industry Corporation, Salem, NH), Version 6.00 .

General procedure for incorporating aspirine and peniciline $G$ into chitosan-cetirizine-based microparticles

First, to optimize the method, pure chitosan microparticles have been made, and then the parameters and working conditions found, have been used and adapted to obtain the chitosan-cetirizine microparticles (CSM.CET and CSL.CET) with aspirin (ASP) and penicillin (PEN). The procedure was performed according to the literature technique [16], with the necessary modifications. Their production was carried out by the method of dripping, under mechanical stirring, a method which requires exact and correct dosing of the active substances. $1 \%$ aqueous solution of sodium tripolyphosphate (TPP) was used as crosslinker.

Microscopic characterization of the obtained microparticles

In order to determine the size and morphology of the obtained microparticles, a microscopic analysis was performed using the FeiQuanta 200F Electronic Scanning Microscope (SEM), which allowed the study of surfaces of the chitosan microparticles to produce clear threedimensional images.

Evaluation of the swelling capacity of the obtained microparticles

According to the literature [17], the therapeutic efficacy is also significantly influenced by the ability of the polymer membranes to retain water; this was tested by determining the degree of inflation at equilibrium. The chitosancetirizine microparticles of penicillin and aspirin of approximately the same size were weighed for dry mass determination $(\mathrm{Wd})$, after which they were left in distilled water at room temperature. Samples were taken every 15 min, dried with filter paper and weighed each time to determine the wet weight (Ww), the operation being repeated until the thermodynamic equilibrium was installed.
The Membrane Swelling Ratio (MSR) is calculated according to the following formula:

$$
\mathrm{MSR} \%=\frac{\mathrm{Ww}-\mathrm{Wd}}{\mathrm{Wd}} * 100,
$$

where:

- Wd is the weight of the dry sample;

-Ww is the weight of the wet sample at thermodynamic equilibrium.

The results were plotted.

In vitro biodegradation of the obtained microparticles

The biodegradability of the microparticles was studied according to the literature using $52975 \mathrm{IU} / \mathrm{mg}$ of lysozyme as the biodegradable agent after it had previously been solubilized at $37^{\circ} \mathrm{C}$ in phosphate buffer $\mathrm{pH} 7.4$ with 10,000 IU / mg. Until the equilibration of the swelling capacity was established, the microparticles were left in the buffer solution ( $p H=7.4$ ), after which they were transferred to the lysozyme buffer and kept in the incubator at $37^{\circ} \mathrm{C}$ for 7 days, the solution being changed daily. On days 1,4 and 7 , the microparticles were removed from the buffer and weighed, following a decrease in mass by biodegradation. The estimation of the percentage of biodegradation ( $D \%)$ was carried out with the following calculation formula:

$$
D \%=\frac{W_{0}-W_{x}}{W_{0} \times 100},
$$

where:

-W is the mass of the microparticles, prior to incubation, at the equilibrium of the swelling capacity;

- Wx is the mass of microparticles after incubation on days 1, 4 and 7 [18].

\section{Results and discussions}

Obtaining and characterization of chitosan-cetirizine derivatives

Chitosan-cetirizine derivatives $(\mathbf{1}, \mathbf{2})$ resulted from an esterification reaction between a hydroxyl group of chitosan and the carboxyl group of cetirizine are presented in figure 1:

By the esterification of the two types of medium and small chitosan, the corresponding derivatives were obtained with cetirizine (1-CSM.CET and 2-CSL.CET). The reaction products were white and homogeneous in appearance, the reaction having a yield of $85.76 \%-96.78 \%$.

By IR spectroscopy, there were highlighted characteristic bands of synthesized compounds, through the glucosamine component (fig. 2):

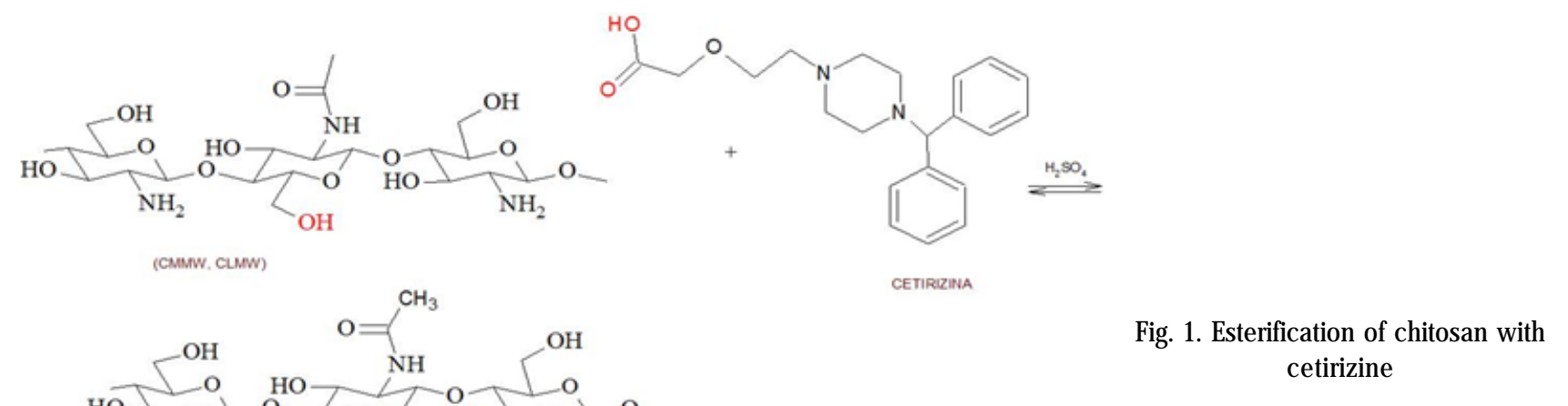




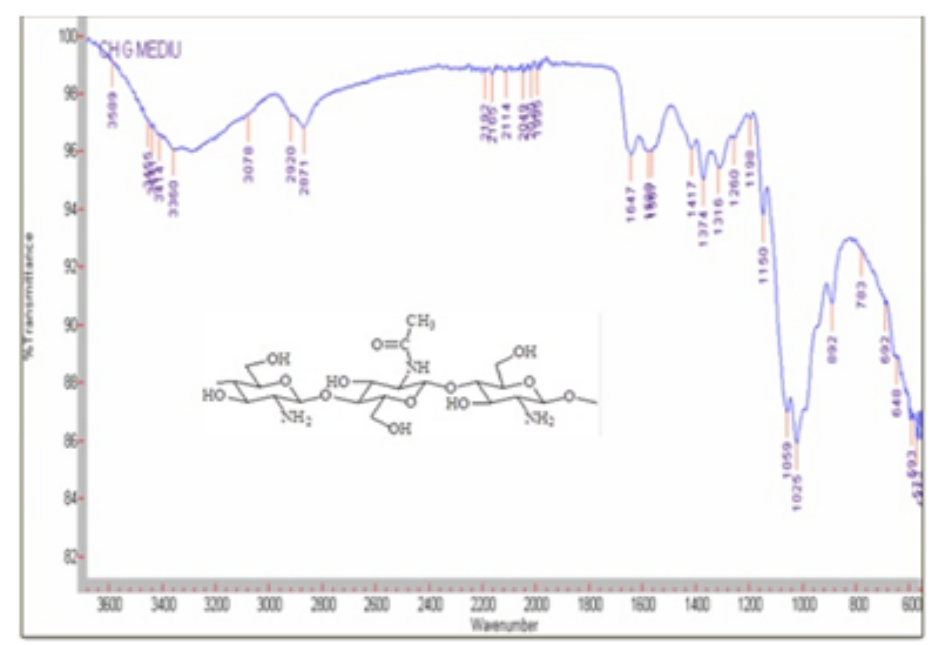

-the carbonyl group $(C=0)$ was highlighted at the wavelength $\lambda=1647 \mathrm{~cm}^{-1}$;

-the amino group (-NH-) exhibits vibrations at $\lambda=1589$ $\mathrm{cm}^{-1}$;

-the hydroxyl alcohol group (-OH) at $\lambda=3300-3400$ $\mathrm{cm}^{-1}$.

Also, the cetirizine component was identified in the spectrum (fig. 3) through:

-the aromatic nucleus is present in the form of multiple bands, the most intense of which were highlighted in the 1539-834 $\mathrm{cm}^{-1}$ region;

-the piperazine component is characterized by a very strong bond of carbon-nitrogen (C-N) in the nucleus at $\lambda=$ $1163 \mathrm{~cm}^{-1}$;

-a methylene group band $\mathrm{CH}_{2}$ at $\lambda=1405 \mathrm{~cm}^{-1}$;

-the presence of the vibration band at $\lambda=1704 \mathrm{~cm}^{-1}$ confirms the realization of the ester-CO bond.

Fig.2. IR spectra of chitosan (glucosamine component highlighting)

\section{Obtaining and SEM image of chitosan-cetirizine microparticles}

The microparticles obtained (fig. 4), after being left stirring for 24 hours at $300 \mathrm{rpm}$, were extracted from the crosslinker solution, washed with distilled water and dried at room temperature (fig. 4).

Following the SEM analysis (fig. 5- CSM.CET-PEN has been taken as an example), both chitosan microparticles with aspirin and penicillin microparticles, have a rough surface of between $400-800 \lambda \mathrm{m}$ in the dry state. The presence of cracks, on their surface, is explained by the high crystallinity of encapsulated substances.

\section{Swelling capacity of the obtained microparticles}

Typically, polysaccharides have a strong water affinity due to the presence of a large number of hydrophilic groups, which can be easily hydrated. The assessment of the

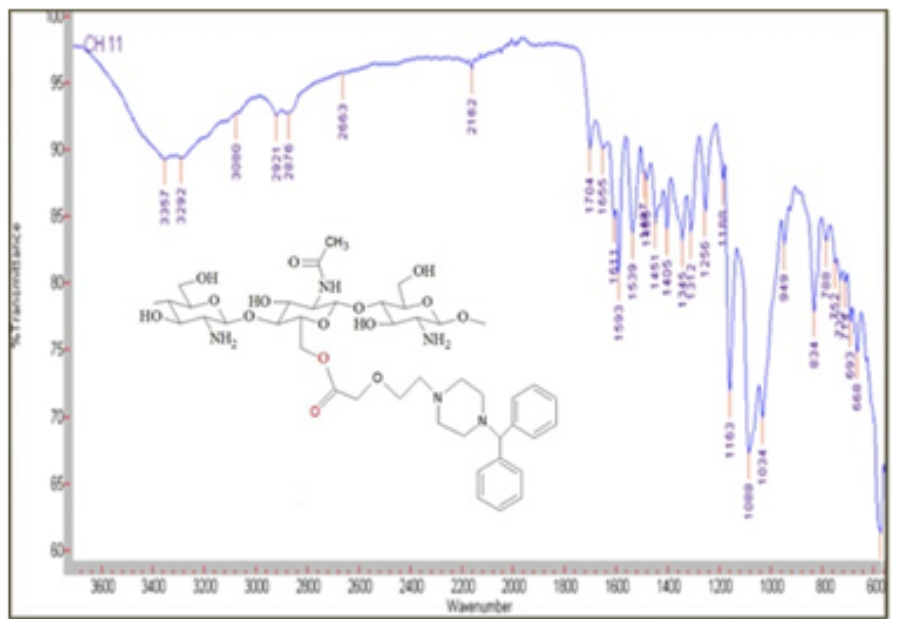

Fig.3. IR spectra of chitosan-cetirizine (cetirizine component highlighting)
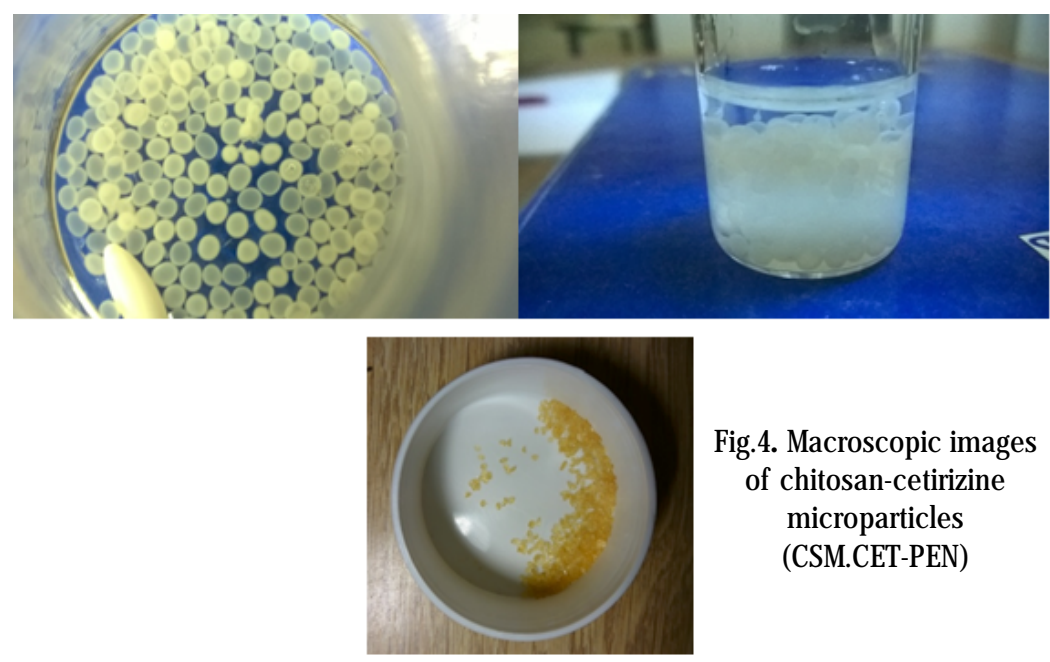

Fig.4. Macroscopic images of chitosan-cetirizine microparticles (CSM.CET-PEN)

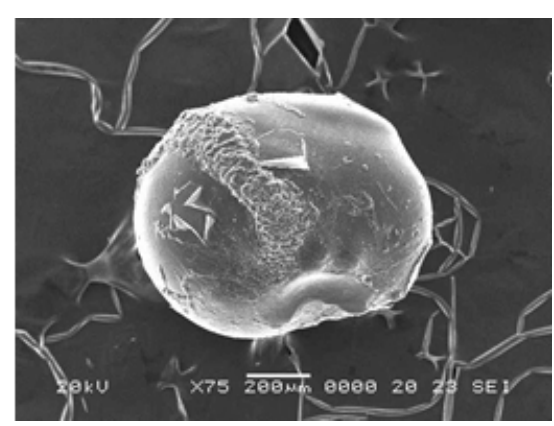

Fig.5. SEM image of chitosan-cetirizine microparticles (CSM.CET-PEN) 


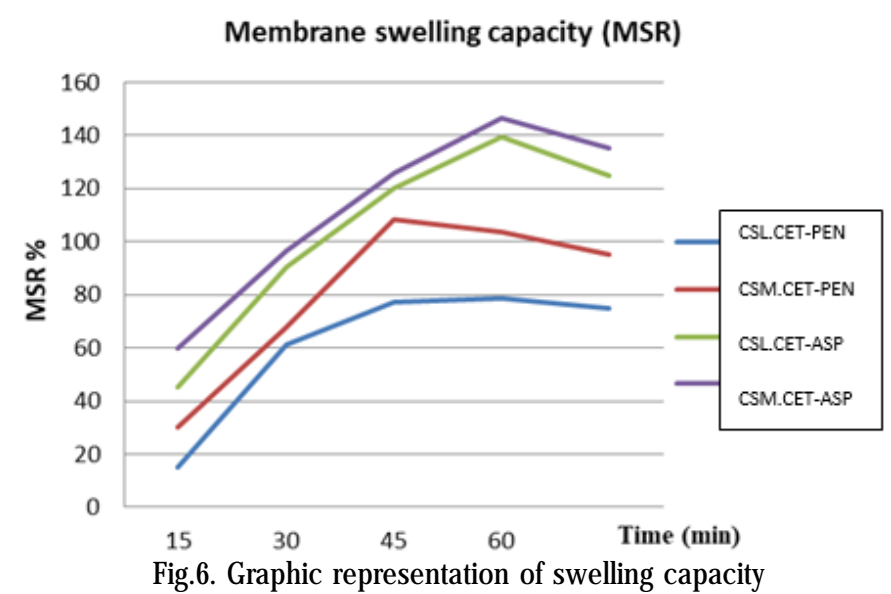

swelling capacity is necessary for analyzing the stability of the microparticles over time.

In the case of low molecular weight chitosan microparticles with penicillin (CSL.CET-PEN), the equilibrium infiltration value is 75\%, calculated after 60 minutes. The highest value was found for CSM.CET+ASP microparticles, namely $135 \%$ and $125 \%$ respectively for CSL.CET-ASP, followed by CSM.CET-PEN microparticles, the swelling capacity being $95 \%$.

\section{In vitro biodegradation of the obtained microparticles}

The in vitro biodegradation of the four microparticles series, under lysozyme activity, was monitored for 7 days, the results being shown in figure 7 .

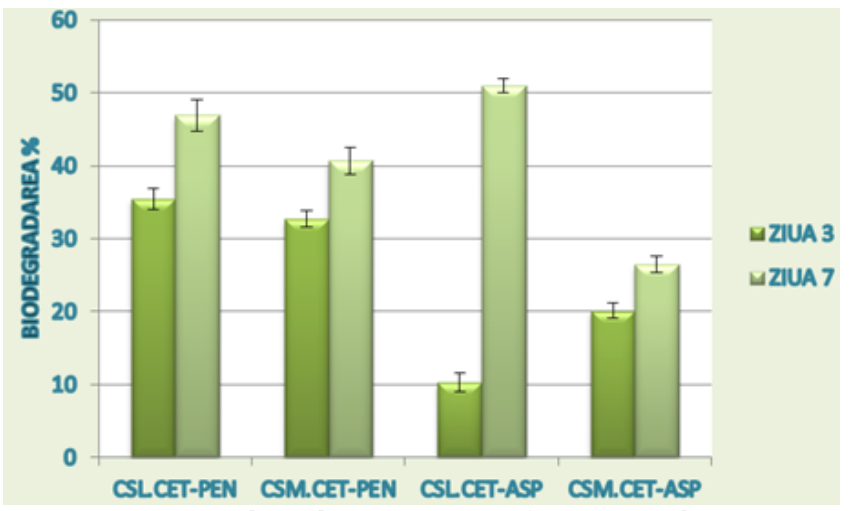

Fig.7. Biodegradation capacity of microparticles

An increase in the seventh-day biodegradation compared to the third day, was found for all derivatives, the highest value being recorded for CSL.CET-ASP, respectively $50.95 \%$ versus $10.28 \%$, on the third day. For medium-weight (CSM.CET-ASP), biodegradation ranges from $20.14 \%$ to $26.45 \%$. On the other hand, in the case of penicillin, for medium-molecular weight chitosan (CSM.CET-PEN), the value increases from $32.71 \%$ (day 3 ) to $40.68 \%$ (day 7 ), while in the case of low molecular weight chitosan derivative (CSL.CET-PEN), a higher biodegradation of $35.45 \%-46.89 \%$ is observed.

\section{Conclusions}

In this paper we have attempted to develop chitosancetirizine microparticles, in which aspirin and penicillin have been incorporated (CSM.CET-PEN, CSL.CET-PEN, CSM.CET-ASP, CSL.CET-ASP), to obtain polymeric matrices able to reduce allergic reactions in patients whose immune system develops extreme sensitivity to a particular active substance.

In this regard, following the determination of the functional characteristics of the obtained compounds, it was observed that synthesis of chitosan-cetirizine derivatives was done properly and efficiently, since IR absorption spectra demonstrated the existence of characteristic vibrations of each functional group: the aromatic ring, the carbonyl group, the carboxyl group, the piperazine moiety and the amino group.

Also, the use of solubilized chitosan in $2 \%$ acetic acid and $1 \%$ TPP, as a crosslinker, resulted in stable, rusty and non-uniform microparticles. In addition, this study confirmed the ability of microparticles to swell by absorbing a percentage of water between 75 and $135 \%$, and to biodegrade to a maximum of $50.95 \%$.

This study has opened the premises for the incorporation of other antibiotics [19] or active substances [20] in order to obtain encapsulated substances with a widespread use in in-hospital infections and beyond.

Acknowledgments: This work was supported by a grant of Ministery of Research and Innovation, CNCS UEFISCDI, project number PN-IIIP1-1.1-PD-2016-0233, within PNCDI III. (Contract No.

PD 144/2018).

\section{References}

1.KATSELOU M, et al. Development and validation of a GC-MS method for thedetermination of hydroxyzine and its active metabolite, cetirizine, inwhole blood. J ournal of Pharmaceutical and Biomedical Analysis 2017, 145: 765-772.

2.PASKO P., et al. Second generation $\mathrm{H} 1$ - antihistamines interaction with food and alcohol-A systematic review. Biomedicine \& Pharmacotherapy 2017, 93: 27-39.

3.HU E., et al. Why are second-generationH1-antihistamines minimally sedating? European J ournal of Pharmacology 2015, 765: 100-106.

4.AMELIAN A, et al. Taste-masking assessment of orally disintegrating tablets and

lyophilisates with cetirizine dihydrochloride microparticles. Saudi Pharmaceutical J ournal 2017, 25: 1144-1150.

5.SANCHEZ-BORGES M, THONG B, BLANCA M, et al. Hypersensitivity reactions to non beta-lactam antimicrobial agents, a statement of the WAO special committee on drug allergy. World Allergy Organization Journal 2013, 6:18.

6.TAMURA A, MIYAWAKI I, YAMADA T, et al. Oral administration of drugs with hypersensitivity potential induces germinal center hyperplasia in secondary lymphoid organ/tissue in Brown Norway rats, and this histological lesion is a promising candidate as a predictive biomarker for drug hypersensitivity occurrence in humans. Toxicology and Applied Pharmacology 2013, 271(1): 30-40.

7.DUMITRIU BUZIA,O., MARDARE, N.,DIACONU, C., The Study of Nystatin Release from Microcapsules Obtained by lonotropic Gelation, Rev.Chim.(Buchares), 67, no.2, 2016, p.232-235

8. DUMITRIU BUZIA.O, DIMA C., DIMA S., Preparation and characterization of chitosan microspheres for vancomycin delivery, Farmacia, 2015, vol. 63, 6,897-902

9.DUMITRIU BUZIA,.O., DIMA, S.,Biopolymer-based Techniques for Encapsulation of Phytochemicals Bioacive in Food and Drug, Mat. Plast., 53, no.1, 2016, p.126-129

10.DRAGOSTIN I, et al. The importance of polymers for encapsulation process and for enhanced cellular functions. Journal of Macromolecular Science, Part A: Pure and Applied Chemistry 2017, 54(7): 489-493.

11.WOLSZLEGER (DRAGAN) M, et al. New thiazolidin-4-ones of ferulic acid with antioxidant potential. Farmacia 2015, 63 (1): 150-154.

12.DRAGAN M, et al. Assessment of in vitro antioxidant and antiinflamatory activities of new azetidin-2-ones derivatives of ferulic acid. Farmacia 2016; 64(5): 717-721.

13.DRAGAN M, etal. Evaluation of anti-inflammatory potential of some new ferulic acid derivatives. Farmacia 2016; 64(2): 194-197.

14.SIONKOW SKA A, PLANECKA A. Preparation and characterization of silk fibroin/ chitosan sponges for tissue engineering. Journal of Molecular Liquids 2013; 178: 5-14. 
15.DUMITRIU RP, et al. Sulfadiazine-Chitosan Conjugates and Their Polyelectrolyte Complexes with Hyaluronate Destined to the Management of Burn Wounds, Materials, 2015, 8(1): 317-338.

16.LUPASCU F, et al. Development, optimization and biological evaluation of chitosan scaffold formulations of new xanthine derivatives for treatment of type-2 diabetes mellitus. European J ournal of Pharmaceutical Sciences 2015; 77, 122-134.

17.DRAGOSTIN OM, et al. New antimicrobial chitosan derivatives for wound dressing applications. Carbohydrate Polymers 2016; 141: 2840.
18.DRAGOSTIN OM, et al. Development and Characterization of Novel Films Based on Sulfonamide-Chitosan Derivatives for Potential Wound Dressing. International J ournal of Molecular Science 2015; 16(12): 29843-29855.

19.DRAGANESCU M, IANCU A.V., FIRESCU D, DUMITRIU B.O., DIACONU C, REBEGEA L, Trends in antimicrobials consumption and antimicrobial resistance in an infectious diseases hospital from the south-eastern ,Farmacia,2016,Vol 64,5,pag.770-774

20.DRAGANESCU, M.,BAROIU, N., BAROIU, L., DIACONU, C., DUMITRIU BUZIA, O., Efficeint administration of human albumin in clostridiumDifficile infection, Rev.Chim. (Bucharest), 68, no.3, 2017,p.602-604

Manuscript received:12.07.2018 